J. Korean Math. Soc. 48 (2011), No. 6, pp. 1269-1283

http://dx.doi.org/10.4134/JKMS.2011.48.6.1269

\title{
MULTIPLE SOLUTIONS FOR QUASILINEAR ELLIPTIC EQUATIONS WITH CRITICAL GROWTH
}

\author{
YOUJUN WANG
}

Abstract. For a class of quasilinear elliptic equations we establish the existence of multiple solutions by variational methods.

\section{Introduction}

In a previous paper (M. J. Alves et al. [1]) the existence of two positive solutions was established for the following equations

$$
-L_{\beta} u+V(x)|u|^{p-2} u=|u|^{q-2} u+g(x), \quad x \in \mathbb{R},
$$

where $L_{\beta} u:=\left[\left|u^{\prime}\right|^{p-2} u^{\prime}\right]^{\prime}+K_{0}\left\{\left[\left(|u|^{\beta}\right)^{\prime}\right]^{p-2}\left(|u|^{\beta}\right)^{\prime}\right\}^{\prime}|u|^{\beta-2} u, K_{0}>0, \beta>1$, $p>1, q \geq p \beta, q>p$ and $g \in L^{s}(\mathbb{R})$ for some $s \geq 1$. In [18], the author considered the equations

$$
-L_{p} u+V(x)|u|^{p-2} u=h(u), \quad x \in \mathbb{R}^{N},
$$

where $L_{p} u:=\Delta_{p} u+\left[\Delta_{p}\left(u^{2}\right)\right] u$ and $\Delta_{p} u:=\operatorname{div}\left(|\nabla u|^{p-2} \nabla u\right)$ is the $p$-Laplacian operator with $1<p<N$. $h(s)$ satisfies subcritical condition. Using the minimax methods, he proved the existence of nontrivial solutions. In [20], the authors studied problem (1.1) when $p=N$ and $h(s)$ satisfies critical condition.

This paper is concerned with the existence of multiple solutions for the quasilinear equations of the form

$$
-L_{p} u=\alpha k(x)|u|^{q-2} u+\beta|u|^{2\left(p^{*}\right)-2} u, \quad x \in \mathbb{R}^{N},
$$

where $1<p<N, p^{*}=N p /(N-p)$ is the critical Sobolev exponent, $2<$ $q<2\left(p^{*}\right)=2 N p /(N-p), k(x) \in L^{r}\left(\mathbb{R}^{N}\right), r=2\left(p^{*}\right) /\left(2\left(p^{*}\right)-q\right) . \alpha$ and $\beta$ are real parameters. We believe that $2\left(p^{*}\right)$ is the critical growth for problem (1.2), since when $p=2$ whose critical exponent is $2\left(2^{*}\right)$ (see $[10,14,19]$ ).

Received July 21, 2010; Revised October 21, 2010.

2010 Mathematics Subject Classification. 35J20, 35J60.

Key words and phrases. quasilinear Schrödinger equations, critical growth, minimax procedure.

Supported by China Post-Doc Science Foundation (No.20100470175). 
Recently, there has been growing interest in the study of the following quasilinear elliptic equations

$$
-\Delta u+V(x) u-\left[\Delta\left(u^{2}\right)\right] u=h(u), \quad x \in \mathbb{R}^{N} .
$$

These equations are related to the existence of standing wave solutions for quasilinear Schrödinger equations of the form

$$
i z_{t}=-\Delta z+V(x) z-l\left(|z|^{2}\right) z-k \Delta g\left(|z|^{2}\right) g^{\prime}\left(|z|^{2}\right) z, \quad x \in \mathbb{R}^{N},
$$

where $V$ is a given potential, $k$ is a real constant, $g$ and $l$ are real functions. Quasilinear Schrödinger equation of this type with $k>0$ arises in various fields of physics, like the theory of superfluids or dissipative quantum mechanics, see e.g. $[13,7]$. Equations with more general dissipative term arise in plasma physics, fluid mechanics, in the theory of Heisenberg ferromagnets, etc. For further physical motivations and a more complete list of references dealing with application, we refer to $[9,10,11]$ and references therein. In [16], the existence of positive ground state solutions for the quasilinear Schrödinger equation $-u^{\prime \prime}+V(x) u-\left(u^{2}\right)^{\prime \prime} u=\theta|u|^{p-1} u$ in $\mathbb{R}$ were constructed as minimizers of a constrained minimization problem, with $\theta$ being the Lagrange multiplier. For the case of subcritical growth, the equation (1.3) were studied by a number of authors. In [10], by a change of variables the quasilinear problem was transformed to a semilinear one and an Orlicz space was used as the working space, and they were able to prove the existence of positive solutions by using the Mountain-pass Theorem. The same method of changing of variables was used in [5], but the usual Sobolev space $H^{1}\left(\mathbb{R}^{N}\right)$ was used as the working space and they proved the existence of a spherically symmetric solution from the classical results given by H. Berestycki and P. L. Lions [8]. The case of the critical growth was also considered recently, among others, by J. M. do Ó [14], A. Moameni [12], Silva [19] and Wang [21].

Our main result is the following:

Theorem 1.1. Suppose that $\Omega:=\left\{x \in \mathbb{R}^{N}: k(x)>0\right\}$ is an open subset of $\mathbb{R}^{N}$ and $0<|\Omega|<+\infty, 2<q<2 p$, Then,

(i) for any $\beta>0$, there exists $\alpha_{1}>0$ such that if $0<\alpha<\alpha_{1}$, the problem (1.2) has a sequence of solutions;

(ii) for any $\alpha>0$, there exists $\beta_{1}>0$ such that if $0<\beta<\beta_{1}$, the problem (1.2) has a sequence of solutions.

The main difficulties to prove Theorem 1.1 are overcoming compactness due to the unboundedness domain $\mathbb{R}^{N}$, the presence of the second order nonhomogeneous term $\left[\Delta_{p}\left(u^{2}\right)\right] u$ and the critical exponent. The approach proposed here is in the sprit of $[10,21]$. By using a changing of variables, the quasilinear equations are reduce to semilinear equations, whose respective associated functionals are well defined. Since the lack of compactness occurs partly due to the appearance of critical exponents and the unboundedness of the domain, both the second concentration-compactness principle due to Lions and 
the concentration-compactness principle at infinity are used to examine the behavior of weakly convergent sequences.

Throughout this paper, $c, c_{1}, c_{2}$ denote positive (possibly different) constants.

\section{Preliminaries}

Define the space $D^{1, p}\left(\mathbb{R}^{N}\right):=\left\{u \in L^{p^{*}}\left(\mathbb{R}^{N}\right): \nabla u \in L^{p}\left(\mathbb{R}^{N}\right)\right\}$, which endowed with the norm $\|u\|=\left(\int_{\mathbb{R}^{N}}|\nabla u|^{p} d x\right)^{1 / p}$.

We note that a major difficulty associated with (1.2) is the natural associated functional $I$ given by

$I(u)=\frac{1}{p} \int_{\mathbb{R}^{N}}\left(1+2^{p-1}|u|^{p}\right)|\nabla u|^{p} d x-\frac{\alpha}{q} \int_{\mathbb{R}^{N}} k(x)|u|^{q} d x-\frac{\beta}{2\left(p^{*}\right)} \int_{\mathbb{R}^{N}}|u|^{2\left(p^{*}\right)} d x$ is not well defined in general, for instance, in $D^{1, p}\left(\mathbb{R}^{N}\right)$. To overcome this difficulty, we employ an argument developed by Colin, Jeanjean in [5] (see also $[10])$. We make the changing of variables $v=f^{-1}(u)$, where $f$ is defined by:

$$
f^{\prime}(t)=\frac{1}{\left(1+2^{p-1}|f(t)|^{p}\right)^{1 / p}}, \quad \text { and } \quad f(0)=0
$$

on $[0, \infty)$ and by $f(t)=-f(-t)$ on $(-\infty, 0]$.

The following results are due to U. Severo [18].

Lemma 2.1. $f$ satisfies the following properties:

$\left(f_{0}\right) f$ is uniquely defined, $C^{2}$ and invertible,

$\left(f_{1}\right)\left|f^{\prime}(t)\right| \leq 1, \quad \forall t \in \mathbb{R}$

$\left(f_{2}\right)|f(t)| \leq|t|, \quad \forall t \in \mathbb{R}$,

$\left(f_{3}\right) \frac{f(t)}{t} \rightarrow 1$ as $t \rightarrow 0$

$\left(f_{4}\right)|f(t)| \leq 2^{1 /(2 p)}|t|^{1 / 2}, \quad \forall t \in \mathbb{R}$,

$\left(f_{5}\right) \frac{1}{2} f(t) \leq t f^{\prime}(t) \leq f(t), \quad \forall t \in \mathbb{R}$ and $t \geq 0$,

$\left(f_{6}\right) \frac{f(t)}{\sqrt{t}} \rightarrow a>0$ as $t \rightarrow \infty$,

$\left(f_{7}\right)$ there exists a positive constant $C$ such that

$$
|f(t)| \geq \begin{cases}C|t|, & |t| \leq 1 \\ C|t|^{1 / 2}, & |t| \geq 1\end{cases}
$$

Therefore, after the change of variables, we can write $I(u)$ as

(2.1) $J(v)=\frac{1}{p} \int_{\mathbb{R}^{N}}|\nabla v|^{p} d x-\frac{\alpha}{q} \int_{\mathbb{R}^{N}} k(x)|f(v)|^{q} d x-\frac{\beta}{2\left(p^{*}\right)} \int_{\mathbb{R}^{N}}|f(v)|^{2\left(p^{*}\right)} d x$.

Lemma 2.2. The functional $J$ is well defined on $D^{1, p}\left(\mathbb{R}^{N}\right)$ and $J \in C^{1}$. Furthermore,

(i) The functional

$$
\mathcal{F}(v):=\int_{\mathbb{R}^{N}} k(x)|f(v)|^{q} d x
$$


is well defined and weakly continuous on $D^{1, p}\left(\mathbb{R}^{N}\right)$. Moreover, $\mathcal{F}(v)$ is continuously differentiable, its derivative $\mathcal{F}^{\prime}(v): D^{1, p}\left(\mathbb{R}^{N}\right) \rightarrow\left(D^{1, p}\left(\mathbb{R}^{N}\right)\right)^{\prime}$ is given by

$$
\left\langle\mathcal{F}^{\prime}(v), h\right\rangle=q \int_{\mathbb{R}^{N}} k(x)|f(v)|^{q-2} f(v) f^{\prime}(v) h d x .
$$

(ii) The functional

$$
\mathcal{G}(v):=\int_{\mathbb{R}^{N}}|f(v)|^{2\left(p^{*}\right)} d x
$$

is well defined. Moreover, $\mathcal{G}(v)$ is continuously differentiable, its derivative $\mathcal{G}^{\prime}(v): D^{1, p}\left(\mathbb{R}^{N}\right) \rightarrow\left(D^{1, p}\left(\mathbb{R}^{N}\right)\right)^{\prime}$ is given by

$$
\left\langle\mathcal{G}^{\prime}(v), h\right\rangle=2\left(p^{*}\right) \int_{\mathbb{R}^{N}}|f(v)|^{2\left(p^{*}\right)-1} f^{\prime}(v) h d x .
$$

Proof. By $\left(f_{3}\right)$ and $\left(f_{6}\right)$, it is clear that $\mathcal{F}$ and $\mathcal{G}$ are well defined on $D^{1, p}\left(\mathbb{R}^{N}\right)$. In order to prove $J \in C^{1}$, it suffices to show both $\mathcal{F}$ and $\mathcal{G}$ have continuous Gâteaux derivative on $D^{1, p}\left(\mathbb{R}^{N}\right)$ (see [22]). We consider only $\mathcal{F}$ since the proof for $\mathcal{G}$ is simpler. Our argument is similar to Lemma 3.10 in [22]. Let $u$, $h \in D^{1, p}\left(\mathbb{R}^{N}\right)$. Given $x \in \mathbb{R}^{N}$ and $0<|t|<1$, by the mean value theorem, there exists $\lambda \in(0,1)$ such that

$$
\begin{aligned}
\frac{\left.|| f(v+t h)\right|^{q}-|f(v)|^{q} \mid}{|t|} & =q|f(v+t \lambda h)|^{q-1}\left|f^{\prime}(v+t \lambda h)\right||h| \\
& \leq c|v+t \lambda h|^{\frac{q-2}{2}}|h| \\
& \leq c\left(|v|^{\frac{q-2}{2}}|h|+|h|^{\frac{q}{2}}\right) .
\end{aligned}
$$

By the Hölder inequality, we have

$$
\int_{\mathbb{R}^{N}} k(x)\left(|v|^{\frac{q-2}{2}}|h|+|h|^{\frac{q}{2}}\right) d x \leq\|k(x)\|_{r}\|h\|_{p^{*}}\left(\|v\|^{\frac{q-2}{2}}+\|h\|^{\frac{q-2}{2}}\right) .
$$

It follows from the Lebesgue dominated convergence theorem that $\mathcal{F}$ is Gâteaux differentiable and

$$
\left\langle\mathcal{F}^{\prime}(v), h\right\rangle=q \int_{\mathbb{R}^{N}} k(x)|f(v)|^{q-2} f(v) f^{\prime}(v) h d x .
$$

Now, we prove continuity of Gâteaux derivative. Assume that $v_{n} \rightarrow v$ in $D^{1, p}\left(\mathbb{R}^{N}\right)$, then $\left|f\left(v_{n}\right)\right|^{2} \rightarrow|f(v)|^{2}$ in $D^{1, p}\left(\mathbb{R}^{N}\right)$. By the continuity of the embedding $D^{1, p}\left(\mathbb{R}^{N}\right) \hookrightarrow L^{p^{*}}\left(\mathbb{R}^{N}\right),\left|f\left(v_{n}\right)\right|^{2} \rightarrow|f(v)|^{2}$ in $L^{p^{*}}\left(\mathbb{R}^{N}\right)$. Let us define $\mathcal{H}(v):=q k(x)|f(v)|^{q-2} f(v) f^{\prime}(v)$, then $\mathcal{H}(v) \in C\left(L^{p^{*}}\left(\mathbb{R}^{N}\right),\left(L^{p^{*}}\left(\mathbb{R}^{N}\right)\right)^{\prime}\right)$ (the proof is analogous to [6] or Theorem A.4 in [22]). It follows that $\mathcal{H}\left(v_{n}\right) \rightarrow \mathcal{H}(v)$ in $\left(L^{p^{*}}\left(\mathbb{R}^{N}\right)\right)^{\prime}$. Using Hölder and Sobolev inequalities, we get

$$
\begin{aligned}
\left\langle\mathcal{F}^{\prime}\left(v_{n}\right)-\mathcal{F}^{\prime}(v), h\right\rangle & \leq\left\|\mathcal{H}\left(v_{n}\right)-\mathcal{H}(v)\right\|_{\left(p^{*}\right)^{\prime}}\|h\|_{p^{*}} \\
& \leq c\left\|\mathcal{H}\left(v_{n}\right)-\mathcal{H}(v)\right\|_{\left(p^{*}\right)^{\prime}}\|h\| .
\end{aligned}
$$

Hence $\left\|\mathcal{F}^{\prime}\left(v_{n}\right)-\mathcal{F}^{\prime}(v)\right\| \rightarrow 0$ and $\mathcal{F} \in C^{1}$. 
As in [18], if $v$ is a nontrivial critical point of $J$, i.e., for all $\omega \in D^{1, p}\left(\mathbb{R}^{N}\right)$,

$$
\begin{aligned}
\left\langle J^{\prime}(v), \omega\right\rangle= & \int_{\mathbb{R}^{N}}|\nabla v|^{p-2} \nabla v \nabla \omega d x-\alpha \int_{\mathbb{R}^{N}} k(x)|f(v)|^{q-2} f(v) f^{\prime}(v) \omega d x \\
& -\beta \int_{\mathbb{R}^{N}}|f(v)|^{2\left(p^{*}\right)-2} f(v) f^{\prime}(v) \omega d x \\
= & 0,
\end{aligned}
$$

we deduce that $v$ is a nontrivial solution of problem

$$
-\Delta_{p} v=g(x, v), \quad x \in \mathbb{R}^{N},
$$

where

$$
g(x, s)=f^{\prime}(s)\left[\alpha k(x)|f(s)|^{q-2} f(s)+\beta|f(s)|^{2\left(p^{*}\right)-2} f(s)\right] .
$$

Then, setting $u=f(v)$ and since $\left(f^{-1}\right)^{\prime}(t)=1 / f^{\prime}\left(f^{-1}\right)=\left(1+2^{p-1}|t|^{p}\right)^{1 / p}$ we conclude that $u$ is a nontrivial solution of the problem (1.2).

The following result is crucial to prove Theorem 1.1 .

Theorem 2.1. Suppose that $\Omega:=\left\{x \in \mathbb{R}^{N}: k(x)>0\right\}$ is an open subset of $\mathbb{R}^{N}$ and $0<|\Omega|<+\infty, 2<q<2 p$, Then,

(i) for any $\beta>0$, there exists $\alpha_{1}>0$ such that if $0<\alpha<\alpha_{1}$, the problem (2.4) has a sequence of solutions $\left\{v_{m}\right\}$ with $J\left(v_{m}\right)<0$ and $J\left(v_{m}\right) \rightarrow 0$ as $m \rightarrow+\infty$;

(ii) for any $\alpha>0$, there exists $\beta_{1}>0$ such that if $0<\beta<\beta_{1}$, the problem (2.4) has a sequence of solutions $\left\{v_{m}\right\}$ with $J\left(v_{m}\right)<0$ and $J\left(v_{m}\right) \rightarrow 0$ as $m \rightarrow+\infty$.

\section{Proof of Theorem 1.1}

First, we recall the second concentration-compactness principle of Lions [8].

Proposition 3.1. Let $\left\{u_{m}\right\}$ be a weakly convergent sequence to $u$ in $D^{1, p}\left(\mathbb{R}^{N}\right)$ such that $\left|u_{m}\right|^{p^{*}} \rightarrow \nu$ and $\left|\nabla u_{m}\right|^{p} \rightarrow \mu$ in the sense of measures. Then, for some at most countable index set $J$,

(i) $\nu=|u|^{p^{*}}+\sum_{j \in J} \nu_{j} \delta_{x_{j}}, \quad \nu_{j}>0$,

(ii) $\mu \geq|\nabla u|^{p}+\sum_{j \in J} \mu_{j} \delta_{x_{j}}, \quad \mu_{j}>0$,

(iii) $S \nu_{j}^{p / p^{*}} \leq \mu_{j}$,

where $S$ is the best Sobolev constant, i.e., $S=\inf \left\{\int_{\mathbb{R}^{N}}|\nabla u|^{p} d x:\|u\|_{p^{*}}^{p^{*}}=1\right\}$, $x_{j} \in \mathbb{R}^{N}, \delta_{x_{j}}$ are Dirac measures at $x_{j}$ and $\mu_{j}, \nu_{j}$ are constants.

The following result can be found in [4].

Proposition 3.2. Let $\left\{u_{m}\right\}$ be a weakly convergent sequence to $u$ in $D^{1, p}\left(\mathbb{R}^{N}\right)$ and define

(i) $\nu_{\infty}=\lim _{R \rightarrow \infty} \limsup _{m \rightarrow \infty} \int_{|x|>R}\left|u_{m}\right|^{p^{*}} d x$,

(ii) $\mu_{\infty}=\lim _{R \rightarrow \infty} \limsup _{m \rightarrow \infty} \int_{|x|>R}\left|\nabla u_{m}\right|^{p} d x$.

Then the quantities $\nu_{\infty}$ and $\mu_{\infty}$ exist and satisfy 
(iii) $\limsup _{m \rightarrow \infty} \int_{\mathbb{R}^{N}}\left|u_{m}\right|^{p^{*}} d x=\int_{\mathbb{R}^{N}} d \nu+\nu_{\infty}, \limsup _{m \rightarrow \infty} \int_{\mathbb{R}^{N}}\left|\nabla u_{m}\right|^{p} d x=\int_{\mathbb{R}^{N}} d \mu+$ $\mu_{\infty}$, and

(iv) $S \nu_{\infty}^{2 / p^{*}} \leq \mu_{\infty}$.

For a real Banach space $E$ and $J \in C^{1}(E, \mathbb{R})$, we say that $J$ satisfies the $(\mathrm{PS})_{c}$ condition if every $(\mathrm{PS})_{c}$ sequence $\left\{v_{n}\right\} \subset E$ possesses a convergent subsequence.

Lemma 3.1. Assume that $2<q<2 p$. Then any $(\mathrm{PS})_{c}$ sequence $\left\{v_{n}\right\}$ is bounded in $D^{1, p}\left(\mathbb{R}^{N}\right)$.

Proof. Let $\left\{v_{n}\right\}$ be a $(\mathrm{PS})_{c}$ sequence, that is,

$$
\begin{aligned}
J\left(v_{n}\right) & =\frac{1}{p} \int_{\mathbb{R}^{N}}\left|\nabla v_{n}\right|^{p} d x-\frac{\alpha}{q} \int_{\mathbb{R}^{N}} k(x)\left|f\left(v_{n}\right)\right|^{q} d x-\frac{\beta}{2\left(p^{*}\right)} \int_{\mathbb{R}^{N}}\left|f\left(v_{n}\right)\right|^{2\left(p^{*}\right)} d x \\
(3.1) & =c+o(1),
\end{aligned}
$$

and for any $\omega \in D^{1, p}\left(\mathbb{R}^{N}\right)$,

$$
\begin{aligned}
\left\langle J^{\prime}\left(v_{n}\right), \omega\right\rangle= & \int_{\mathbb{R}^{N}}\left|\nabla v_{n}\right|^{p-2} \nabla v_{n} \nabla \omega d x-\alpha \int_{\mathbb{R}^{N}} k(x)\left|f\left(v_{n}\right)\right|^{q-2} f\left(v_{n}\right) f^{\prime}\left(v_{n}\right) \omega d x \\
& -\beta \int_{\mathbb{R}^{N}}\left|f\left(v_{n}\right)\right|^{2\left(p^{*}\right)-1} f^{\prime}\left(v_{n}\right) \omega d x \\
(3.2) \quad & o(1)\|\omega\| .
\end{aligned}
$$

Choose $\omega=\omega_{n}=\sqrt[p]{1+2^{p-1}\left|f\left(v_{n}\right)\right|^{p}} f\left(v_{n}\right)$, then

$$
\left|\nabla \omega_{n}\right|=\left(1+\frac{2^{p-1}\left|f\left(v_{n}\right)\right|^{p}}{1+2^{p-1}\left|f\left(v_{n}\right)\right|^{p}}\right)\left|\nabla v_{n}\right|,
$$

thus $\left\|\omega_{n}\right\| \leq c\left\|v_{n}\right\|$. In particular, recording that $\left\{v_{n}\right\}$ is a $(\mathrm{PS})_{c}$ consequence,

$$
\begin{aligned}
\left\langle J^{\prime}\left(v_{n}\right), \omega_{n}\right\rangle= & \int_{\mathbb{R}^{N}}\left(1+\frac{2^{p-1}\left|f\left(v_{n}\right)\right|^{p}}{1+2^{p-1}\left|f\left(v_{n}\right)\right|^{p}}\right)\left|\nabla v_{n}\right|^{p} d x-\alpha \int_{\mathbb{R}^{N}} k(x)\left|f\left(v_{n}\right)\right|^{q} d x \\
& -\beta \int_{\mathbb{R}^{N}}\left|f\left(v_{n}\right)\right|^{2\left(p^{*}\right)} d x \\
= & o(1)\left\|v_{n}\right\| .
\end{aligned}
$$

By Hölder and Sobolev inequalities, we have

$$
\left.\left.\int_{\mathbb{R}^{N}}|\nabla| f\left(v_{n}\right)\right|^{2}\right|^{p} d x=\int_{\mathbb{R}^{N}} \frac{2^{p}\left|f\left(v_{n}\right)\right|^{p}}{1+2^{p-1}\left|f\left(v_{n}\right)\right|^{p}}\left|\nabla v_{n}\right|^{p} d x \leq 2 \int_{\mathbb{R}^{N}}\left|\nabla v_{n}\right|^{p} d x .
$$

Therefore,

$$
\begin{aligned}
c+o(1) & =J\left(v_{n}\right)-\frac{1}{2\left(p^{*}\right)}\left\langle J^{\prime}\left(v_{n}\right), \omega_{n}\right\rangle \\
& =\int_{\mathbb{R}^{N}}\left[\frac{1}{p}-\frac{1}{2\left(p^{*}\right)}\left(1+\frac{2^{p-1}\left|f\left(v_{n}\right)\right|^{p}}{1+2^{p-1}\left|f\left(v_{n}\right)\right|^{p}}\right)\right]\left|\nabla v_{n}\right|^{2} d x
\end{aligned}
$$




$$
\begin{aligned}
& -\frac{\alpha}{r} \int_{\mathbb{R}^{N}} k(x)\left|f\left(v_{n}\right)\right|^{q} d x \\
\geq & \frac{1}{N} \int_{\mathbb{R}^{N}}\left|\nabla v_{n}\right|^{p} d x-\frac{\alpha}{r}\left(\int_{\mathbb{R}^{N}}|k(x)|^{r} d x\right)^{\frac{1}{r}}\left(\int_{\mathbb{R}^{N}}\left|f\left(v_{n}\right)\right|^{2\left(p^{*}\right)} d x\right)^{\frac{q}{2\left(p^{*}\right)}} \\
\geq & \frac{1}{N} \int_{\mathbb{R}^{N}}\left|\nabla v_{n}\right|^{p} d x-\frac{\alpha}{r}\left(\int_{\mathbb{R}^{N}}|k(x)|^{r} d x\right)^{\frac{1}{r}}\left(\left.\left.\int_{\mathbb{R}^{N}}|\nabla| f\left(v_{n}\right)\right|^{2}\right|^{p} d x\right)^{\frac{q}{2 p}} \\
\geq & \frac{1}{N}\left\|v_{n}\right\|^{p}-c\left\|v_{n}\right\|^{\frac{q}{2}}
\end{aligned}
$$

which imply that $\left\{v_{n}\right\}$ is bounded since $q<2 p$.

The following result in [15] will be useful in the sequel.

Lemma 3.2. Let $x, y \in \mathbb{R}^{N}$ and $\langle\cdot, \cdot\rangle$ be the standard scalar product in $\mathbb{R}^{N}$. Then

$$
\left\langle|x|^{p-2} x-|y|^{p-2} y, x-y\right\rangle \geq \begin{cases}c|x-y|^{p}, & p \geq 2, \\ c \frac{|x-y|^{2}}{(|x|+|y|)^{2-p}}, & 1<p<2 .\end{cases}
$$

Proposition 3.3. Assume $2<q<2 p$ and $c<0$.

(i) For any $\beta>0$, there exists $\alpha_{1}>0$ such that if $0<\alpha<\alpha_{1}$, then $J$ satisfies $(\mathrm{PS})_{c}$.

(ii) For any $\alpha>0$, there exists $\beta_{1}>0$ such that if $0<\beta<\beta_{1}$, then $J$ satisfies $(\mathrm{PS})_{c}$.

Proof. Let $\left\{v_{n}\right\}$ be a $(\mathrm{PS})_{c}$ sequence, by Lemma 3.1, $\left\{v_{n}\right\}$ is bounded in $D^{1, p}\left(\mathbb{R}^{N}\right)$. It is easy to check that $\left\{\left|f\left(v_{n}\right)\right|^{2}\right\}$ is also bounded in $D^{1, p}\left(\mathbb{R}^{N}\right)$.

We can assume, going if necessary to a subsequence, that $v_{n} \rightarrow v$ in $D^{1, p}\left(\mathbb{R}^{N}\right)$, $v_{n} \rightarrow v$ a.e. in $\mathbb{R}^{N}$. Since $f \in C^{2},\left|f\left(v_{n}\right)\right|^{2} \rightarrow|f(v)|^{2}$ a.e. in $\mathbb{R}^{N}$. Therefore, $\left|f\left(v_{n}\right)\right|^{2} \rightarrow|f(v)|^{2}$ in $D^{1, p}\left(\mathbb{R}^{N}\right)$. By Proposition 3.1, there exist measures $\mu$ and $\nu$ such that $\left.\left.|\nabla| f\left(v_{n}\right)\right|^{2}\right|^{p} \rightarrow \mu$ and $\left|f\left(v_{n}\right)\right|^{2\left(p^{*}\right)} \rightarrow \nu$. Let $x_{k}$ be a singular point of the measures $\mu$ and $\nu$. We define a function $\phi \in C_{0}^{\infty}\left(\mathbb{R}^{N}\right)$ such that $\phi(x)=1$ in $B\left(x_{j}, \epsilon\right), \phi(x)=0$ in $\mathbb{R}^{n} \backslash B\left(x_{j}, 2 \epsilon\right)$ and $|\nabla \phi| \leq 2 / \epsilon$ in $\mathbb{R}^{N}$. Let $\tilde{\omega}_{n}=\sqrt[p]{1+2^{p-1}\left|f\left(v_{n}\right)\right|^{p}} f\left(v_{n}\right) \phi$, then $\left\{\tilde{\omega}_{n}\right\}$ is bounded in $D^{1, p}\left(\mathbb{R}^{N}\right)$. We have $\lim _{n \rightarrow \infty}\left\langle J^{\prime}\left(v_{n}\right), \tilde{\omega}_{n}\right\rangle=0$, i.e.,

$$
\begin{aligned}
\lim _{n \rightarrow \infty} & \int_{\mathbb{R}^{N}} \sqrt[p]{1+2^{p-1}\left|f\left(v_{n}\right)\right|^{p}} f\left(v_{n}\right)\left|\nabla v_{n}\right|^{p-2} \nabla v_{n} \nabla \phi d x \\
= & \lim _{n \rightarrow \infty}\left[\int_{\mathbb{R}^{N}}\left(1+\frac{2^{p-1}\left|f\left(v_{n}\right)\right|^{p}}{1+2^{p-1}\left|f\left(v_{n}\right)\right|^{p}}\right)\left|\nabla v_{n}\right|^{p} \phi d x\right. \\
& \left.-\alpha \int_{\mathbb{R}^{N}} k(x)\left|f\left(v_{n}\right)\right|^{q} \phi d x-\beta \int_{\mathbb{R}^{N}}\left|f\left(v_{n}\right)\right|^{2\left(p^{*}\right)} \phi d x\right] .
\end{aligned}
$$

By $\left(f_{5}\right)$,

$$
\left.\lim _{n \rightarrow \infty}\left|\int_{\mathbb{R}^{N}} \sqrt[p]{1+2^{p-1}\left|f\left(v_{n}\right)\right|^{p}} f\left(v_{n}\right)\right| \nabla v_{n}\right|^{p-2} \nabla v_{n} \nabla \phi d x \mid
$$




$$
\begin{aligned}
& \leq \lim _{n \rightarrow \infty} c \int_{\mathbb{R}^{N}}\left|v_{n}\right|\left|\nabla v_{n}\right|^{p-1}|\nabla \phi| d x \\
& \leq c \lim _{n \rightarrow \infty}\left[\left(\int_{\mathbb{R}^{N}}\left|\nabla v_{n}\right|^{p} d x\right)^{(p-1) / p}\left(\int_{\mathbb{R}^{N}}\left|v_{n} \nabla \phi\right|^{p} d x\right)^{1 / p}\right] \\
& \leq c\left(\int_{\mathbb{R}^{N}}|v \nabla \phi|^{p} d x\right)^{1 / p} \\
& \leq c\left(\int_{B\left(x_{j}, 2 \epsilon\right)}|v|^{p^{*}} d x\right)^{1 / p^{*}} \rightarrow 0 \text { as } \epsilon \rightarrow 0 .
\end{aligned}
$$

Then by the weak continuity of $\mathcal{F}(v)$,

$$
\begin{aligned}
0= & \lim _{n \rightarrow \infty}\left[\int_{\mathbb{R}^{N}}\left(1+\frac{2^{p-1}\left|f\left(v_{n}\right)\right|^{p}}{1+2^{p-1}\left|f\left(v_{n}\right)\right|^{p}}\right)\left|\nabla v_{n}\right|^{p} \phi d x-\alpha \int_{\mathbb{R}^{N}} k(x)\left|f\left(v_{n}\right)\right|^{q} \phi d x\right. \\
& \left.-\beta \int_{\mathbb{R}^{N}}\left|f\left(v_{n}\right)\right|^{2\left(p^{*}\right)} \phi d x\right] \\
\geq & \lim _{n \rightarrow \infty}\left[\left.\left.\frac{1}{2} \int_{\mathbb{R}^{N}}|\nabla| f\left(v_{n}\right)\right|^{2}\right|^{p} \phi d x-\alpha \int_{\mathbb{R}^{N}} k(x)\left|f\left(v_{n}\right)\right|^{q} \phi d x\right. \\
& \left.-\beta \int_{\mathbb{R}^{N}}\left|f\left(v_{n}\right)\right|^{2\left(p^{*}\right)} \phi d x\right] \\
= & \frac{\mu_{j}}{2}-\beta \nu_{j} \quad \text { as } \epsilon \rightarrow 0 .
\end{aligned}
$$

Hence $2 \beta \nu_{j} \geq \mu_{j}$. Combining with (iii) of Proposition 3.1 we have that either

$$
\text { (I) } \nu_{j}=0, \quad \text { or } \quad \text { (II) } \nu_{j} \geq\left(2^{-1} \beta^{-1} S\right)^{N / p} \text {. }
$$

To obtain the possible concentration of mass at infinity, we define a cut off function $\phi_{R} \in C_{0}^{\infty}\left(\mathbb{R}^{N}\right)$ such that $\phi_{R}(x)=0$ on $|x|<R$ and $\phi_{R}(x)=1$ on $|x|>R+1$. Since $\left\{\phi_{R} \omega_{n}\right\}$ is bounded in $D^{1, p}\left(\mathbb{R}^{N}\right)$, we have

$$
\begin{aligned}
\lim _{n \rightarrow \infty} \int_{\mathbb{R}^{N}} \sqrt[p]{1+2^{p-1}\left|f\left(v_{n}\right)\right|^{p}} f\left(v_{n}\right)\left|\nabla v_{n}\right|^{p-2} \nabla v_{n} \nabla \phi_{R} d x \\
=\lim _{n \rightarrow \infty}\left[\int_{\mathbb{R}^{N}}\left(1+\frac{2^{p-1}\left|f\left(v_{n}\right)\right|^{p}}{1+2^{p-1}\left|f\left(v_{n}\right)\right|^{p}}\right)\left|\nabla v_{n}\right|^{p} \phi_{R} d x\right. \\
\left.\quad-\alpha \int_{\mathbb{R}^{N}} k(x)\left|f\left(v_{n}\right)\right|^{q} \phi_{R} d x-\beta \int_{\mathbb{R}^{N}}\left|f\left(v_{n}\right)\right|^{2\left(p^{*}\right)} \phi_{R} d x\right] .
\end{aligned}
$$

Notice that

$$
\left.\limsup _{R \rightarrow \infty} \limsup _{n \rightarrow \infty}\left|\int_{\mathbb{R}^{N}} \sqrt[p]{1+2^{p-1}\left|f\left(v_{n}\right)\right|^{p}} f\left(v_{n}\right)\right| \nabla v_{n}\right|^{p-2} \nabla v_{n} \nabla \phi_{R} d x \mid=0 .
$$

By the weak continuity of $\mathcal{F}$, we have

$$
\left.\lim _{n \rightarrow \infty}\left|\int_{|x|>R} k(x)\right| f\left(v_{n}\right)\right|^{q} \phi_{R} d x \mid \rightarrow 0 \quad \text { as } R \rightarrow \infty .
$$


Therefore,

$$
\begin{aligned}
0=\lim _{n \rightarrow \infty}[ & \int_{\mathbb{R}^{N}}\left(1+\frac{2^{p-1}\left|f\left(v_{n}\right)\right|^{p}}{1+2^{p-1}\left|f\left(v_{n}\right)\right|^{p}}\right) \phi_{R}\left|\nabla v_{n}\right|^{p} d x-\alpha \int_{\mathbb{R}^{N}} k(x)\left|f\left(v_{n}\right)\right|^{q} \phi_{R} d x \\
& \left.\quad-\beta \int_{\mathbb{R}^{N}}\left|f\left(v_{n}\right)\right|^{2\left(p^{*}\right)} \phi_{R} d x\right] \\
\geq \lim _{n \rightarrow \infty}[ & {\left[\left.\left.\frac{1}{2} \int_{\mathbb{R}^{N}} \phi_{R}|\nabla| f\left(v_{n}\right)\right|^{2}\right|^{p} d x-\alpha \int_{\mathbb{R}^{N}} k(x)\left|f\left(v_{n}\right)\right|^{q} \phi_{R} d x\right.} \\
& \left.\quad-\beta \int_{\mathbb{R}^{N}}\left|f\left(v_{n}\right)\right|^{2\left(p^{*}\right)} \phi_{R} d x\right] \\
= & \frac{\mu_{\infty}}{2}-\beta \nu_{\infty} \quad \text { as } R \rightarrow \infty .
\end{aligned}
$$

Combining with (iv) of Proposition 3.2 we have that either

$$
\text { (III) } \nu_{\infty}=0, \quad \text { or } \quad(\mathrm{IV}) \nu_{\infty} \geq\left(2^{-1} \beta^{-1} S\right)^{N / p} \text {. }
$$

Now we claim that (II) and (IV) cannot occur if $\alpha$ and $\beta$ are chosen properly. Indeed, from the weak lower semicontinuity of the norm and the weak continuity of $\mathcal{F}$,

$$
\begin{aligned}
& 0>c= \lim _{n \rightarrow \infty}\left(J\left(v_{n}\right)-\frac{1}{2\left(p^{*}\right)}\left\langle J^{\prime}\left(v_{n}\right), \sqrt[p]{1+2^{p-1}\left|f\left(v_{n}\right)\right|^{p}} f\left(v_{n}\right)\right\rangle\right) \\
&= \lim _{n^{\prime} \rightarrow \infty}\left(\int_{\mathbb{R}^{N}}\left[\frac{1}{p}-\frac{1}{2\left(p^{*}\right)}\left(1+\frac{2^{p-1}\left|f\left(v_{n}\right)\right|^{p}}{1+2^{p-1}\left|f\left(v_{n}\right)\right|^{p}}\right)\right]\left|\nabla v_{n}\right|^{p} d x\right. \\
&\left.\quad-\alpha\left(\frac{1}{q}-\frac{1}{2\left(p^{*}\right)}\right) \int_{\mathbb{R}^{N}} k(x)\left|f\left(v_{n}\right)\right|^{p} d x\right) \\
& \geq \lim _{n \rightarrow \infty}\left(\frac{1}{N} \int_{\mathbb{R}^{N}}\left|\nabla v_{n}\right|^{p} d x-\frac{\alpha}{q r}\|k(x)\|_{r}\left\|\left|f\left(v_{n}\right)\right|^{2}\right\|_{p^{*}}^{\frac{q}{2}}\right) \\
& \geq \frac{1}{N} \int_{\mathbb{R}^{N}}|\nabla v|^{p} d x-\frac{\alpha}{q r}\|k(x)\|_{r}\left\|\left|f\left(v_{n}\right)\right|^{2}\right\|_{p^{*}}^{\frac{q}{2}} \\
& \geq\left.\left.\frac{1}{2 N} \int_{\mathbb{R}^{N}}|\nabla| f(v)\right|^{2}\right|^{p} d x-\frac{\alpha}{q r}\|k(x)\|_{r}\left\||f(v)|^{2}\right\|_{p^{*}}^{\frac{q}{2}} \\
& \geq \frac{S}{2 N}\left\||f(v)|^{2}\right\|_{p^{*}}^{p}-\frac{\alpha}{q r}\|k(x)\|_{r}\left\||f(v)|^{2}\right\|_{p^{*}}^{\frac{q}{2}},
\end{aligned}
$$

which yields $\left\||f(v)|^{2}\right\|_{p^{*}} \leq c \alpha^{\frac{2}{2 p-q}}$. Therefore,

$$
\begin{gathered}
0>c=\lim _{n \rightarrow \infty}\left(J\left(v_{n}\right)-\frac{1}{2\left(p^{*}\right)}\left\langle J^{\prime}\left(v_{n}\right), \sqrt[p]{1+2^{p-1}\left|f\left(v_{n}\right)\right|^{p}} f\left(v_{n}\right)\right\rangle\right) \\
=\lim _{n \rightarrow \infty}\left(\int_{\mathbb{R}^{N}}\left[\frac{1}{p}-\frac{1}{2\left(p^{*}\right)}\left(1+\frac{2^{p-1}\left|f\left(v_{n}\right)\right|^{p}}{1+2^{p-1}\left|f\left(v_{n}\right)\right|^{p}}\right)\right]\left|\nabla v_{n}\right|^{p} d x\right. \\
\left.-\left.\alpha\left(\frac{1}{p}-\frac{1}{2\left(p^{*}\right)}\right) \int_{\mathbb{R}^{N}} k(x)|| f\left(v_{n}\right)\right|^{q} d x\right)
\end{gathered}
$$




$$
\begin{aligned}
& \geq \frac{1}{N} \lim _{R \rightarrow \infty} \lim _{n \rightarrow \infty} \int_{\mathbb{R}^{N}}\left|\nabla v_{n}\right|^{p} \phi_{R} d x-\frac{\alpha}{q r}\|k(x)\|_{r}\left\|\left|f\left(v_{n}\right)\right|^{2}\right\|_{p^{*}}^{q / 2} \\
& \geq \frac{1}{2 N} \mu_{\infty}-c \alpha^{q /(2 p-q)} \\
& \geq \frac{1}{2 N} 2^{(p-N) / p} \beta^{(p-N) / p} S^{N / p}-c \alpha^{q /(2 p-q)} .
\end{aligned}
$$

However, if $\alpha>0$ is given, we can choose $\beta_{1}$ so small such that for every $0<\beta<\beta_{1}$, the last term on the right-hand side above is greater than 0 , which is a contradiction. Similarly, if $\beta>0$ is given, we can take $\alpha_{1}$ so small such that for every $0<\alpha<\alpha_{1}$, the last term on the right-hand side above is greater than 0. Analogously, we can prove that (II) cannot occur for each $j$. Thus,

$$
\int_{\mathbb{R}^{N}}\left|f\left(v_{n}\right)\right|^{2\left(p^{*}\right)} d x \rightarrow \int_{\mathbb{R}^{N}}|f(v)|^{2\left(p^{*}\right)} d x \quad \text { as } n \rightarrow \infty .
$$

In order to prove $v_{n} \rightarrow v$ in $D^{1, p}\left(\mathbb{R}^{N}\right)$, we adapt some arguments in [3]. Define functions

$$
\tau_{\eta}(s)= \begin{cases}s, & \text { if }|s| \leq \eta, \\ \eta \frac{s}{|s|}, & \text { if }|s|>\eta .\end{cases}
$$

Fix a compact set $K \subset \mathbb{R}^{N}$ and take a cut-off function $\phi_{K}: \mathbb{R}^{N} \rightarrow \mathbb{R}$, satisfying:

$$
\phi_{K} \in C_{0}^{\infty}\left(\mathbb{R}^{N}\right), \quad 0 \leq \phi_{K} \leq 1, \quad \text { and } \phi_{K}(x)=1 \quad \text { on } K
$$

Then $\phi_{K} \tau_{\eta}\left(f\left(v_{n}\right)-f(v)\right) \in D^{1, p}\left(\mathbb{R}^{N}\right)$. Since $\left\{v_{n}\right\}$ is a $(\mathrm{PS})_{c}$ sequence, we have

$$
\begin{aligned}
o(1)= & \left\langle J^{\prime}\left(v_{n}\right)-J^{\prime}(v), \phi_{K} \tau_{\eta}\left(f\left(v_{n}\right)-f(v)\right)\right\rangle \\
= & \int_{\mathbb{R}^{N}}\left(\left|\nabla v_{n}\right|^{p-2} \nabla v_{n}-|\nabla v|^{p-2} \nabla v\right) \nabla\left[\phi_{K} \tau_{\eta}\left(f\left(v_{n}\right)-f(v)\right)\right] d x \\
& -\alpha \int_{\mathbb{R}^{N}} k(x)\left[\left|f\left(v_{n}\right)\right|^{q-2} f\left(v_{n}\right) f^{\prime}\left(v_{n}\right)-|f(v)|^{q-2} f(v) f^{\prime}(v)\right] \\
& \cdot\left[\phi_{K} \tau_{\eta}\left(f\left(v_{n}\right)-f(v)\right)\right] d x \\
& -\beta \int_{\mathbb{R}^{N}}\left[\left|f\left(v_{n}\right)\right|^{2\left(p^{*}\right)-2} f\left(v_{n}\right) f^{\prime}\left(v_{n}\right)-|f(v)|^{2\left(p^{*}\right)-2} f(v) f^{\prime}(v)\right] \\
& \cdot\left[\phi_{K} \tau_{\eta}\left(f\left(v_{n}\right)-f(v)\right)\right] d x .
\end{aligned}
$$

We denote the three integrations on the right-hand of (3.6) by $I_{1}, I_{2}$ and $I_{3}$. By (3.5), we have $\left|I_{2}\right| \leq c \eta$ and $\left|I_{3}\right| \leq c \eta$. Thus, by (3.6), we conclude that $\left|I_{1}\right| \leq c \eta$.

Let $e_{n}:=\left\langle\left|\nabla v_{n}\right|^{p-2} \nabla v_{n}-|\nabla v|^{p-2} \nabla v, \nabla\left[\phi_{K} \tau_{\eta}\left(f\left(v_{n}\right)-f(v)\right)\right]\right\rangle$. By using Lemma 3.2, a direct calculation show that $e_{n} \geq 0$. Take $0<\theta<1$ and split $K$ into

$$
S_{n}^{\eta}=\left\{x \in K ;\left|f\left(v_{n}\right)-f(v)\right| \leq \eta\right\}, \quad G_{n}^{\eta}=\left\{x \in K ;\left|f\left(v_{n}\right)-f(v)\right|>\eta\right\} .
$$


Then

$$
\begin{aligned}
\int_{\mathbb{R}^{N}} e_{n}^{\theta} d x & =\int_{S_{n}^{\eta}} e_{n}^{\theta} d x+\int_{G_{n}^{\eta}} e_{n}^{\theta} d x \\
& \leq\left(\int_{S_{n}^{\eta}} e_{n} d x\right)^{\theta}\left|S_{n}^{\eta}\right|^{1-\theta}+\left(\int_{G_{n}^{\eta}} e_{n} d x\right)^{\theta}\left|G_{n}^{\eta}\right|^{1-\theta} .
\end{aligned}
$$

Now, fixed $k$, then $\left|G_{n}^{\eta}\right| \rightarrow 0$ as $n \rightarrow \infty$ and from the uniform boundedness in $L^{1}$ we get

$$
\limsup _{n \rightarrow \infty} \int_{\mathbb{R}^{N}} e_{n}^{\theta} d x \leq(C k)^{\theta}|K|^{1-\theta}
$$

Let $k \rightarrow 0$, we get that $e_{n}^{\theta} \rightarrow 0$ in $L^{1}$. By Lemma 3.2 , we have $\nabla v_{n} \rightarrow \nabla v$ in $L^{\tau}\left(\mathbb{R}^{N}\right)$ for $1<\tau<p$. By passing to a subsequence, we have $\nabla v_{n} \rightarrow \nabla v$ a.e. in $\mathbb{R}^{N}$. It follows that $v_{n} \rightarrow v$ in $D^{1, p}\left(\mathbb{R}^{N}\right)$. This completes the proof of Proposition 3.5.

Now we use minimax procedure to prove Theorem 2.1, then Theorem 1.1 is a consequence of it. For a Banach space $X$, we define the set

$\Sigma=\{A \subset X \backslash\{0\}: A$ is closed in $X$ and symmetric with respect to the origin $\}$.

For $A \in \Sigma$, define

$$
\gamma(A):=\inf \left\{m \in \mathbb{N} ; \exists \varphi \in C\left(A, \mathbb{R}^{m} \backslash\{0\}\right), \varphi(x)=-\varphi(-x)\right\},
$$

if there is no mapping $\varphi$ as above for any $m \in \mathbb{N}$, then $\gamma(A):=+\infty$. We refer to the properties of the genus in [17].

Proposition 3.4. Let $A, B \in X$.

(1) If there exists an odd map $f \in C(A, B)$, then $\gamma(A) \leq \gamma(B)$,

(2) if $A \subset B$, then $\gamma(A) \leq \gamma(B)$,

(3) $\gamma(A \cup B) \leq \gamma(A)+\gamma(B)$,

(4) if $A$ is compact, there exists a symmetric neighborhood $N$ of $A$, such that $\gamma(\bar{N})=\gamma(A)$.

Let $J$ be the functional defined above, then

$$
\begin{aligned}
J(v)= & \frac{1}{p} \int_{\mathbb{R}^{N}}|\nabla v|^{p} d x-\frac{\alpha}{q} \int_{\mathbb{R}^{N}} k(x)|f(v)|^{q} d x-\frac{\beta}{2\left(p^{*}\right)} \int_{\mathbb{R}^{N}}|f(v)|^{2\left(p^{*}\right)} d x \\
\geq & \frac{1}{p}\|v\|^{p}-\frac{\alpha}{q}\left(\int_{\mathbb{R}^{N}}|k(x)|^{r} d x\right)^{1 / r}\left(\int_{\mathbb{R}^{N}}|f(v)|^{2\left(p^{*}\right)} d x\right)^{q / 2\left(p^{*}\right)} \\
& -\frac{\beta}{2\left(p^{*}\right)} \int_{\mathbb{R}^{N}}|f(v)|^{2\left(p^{*}\right)} d x \\
\geq & \frac{1}{p}\|v\|^{p}-\alpha c_{1}\|v\|^{q / 2}-\beta c_{2}\|v\|^{p^{*}} .
\end{aligned}
$$

We define the function

$$
Q(t):=\frac{1}{p} t^{p}-\alpha c_{1} t^{q / 2}-\beta c_{2} t^{p^{*}},
$$


then it is easy to see that for given $\beta>0$, there exists $\alpha_{2}>0$ so small that for every $0<\alpha<\alpha_{2}$, there exists $0<T_{0}<T_{1}$ such that $Q(t)<0$ for $0<t<T_{0}$, $Q(t)>0$ for $T_{0}<t<T_{1}$ and $Q(t)<0$ for $t>T_{1}$. Analogously, for given $\alpha>0$, we can choose $\beta_{2}>0$ with the property that $T_{0}, T_{1}$ as above for each $0<\beta<\beta_{2}$. Clearly, $Q\left(T_{0}\right)=Q\left(T_{1}\right)=0$. Using the same idea as in [2], we consider the truncated functional

$$
\tilde{J}(v)=\frac{1}{p} \int_{\mathbb{R}^{N}}|\nabla v|^{p} d x-\frac{\alpha}{q} \int_{\mathbb{R}^{N}} k(x)|f(v)|^{q} d x-\frac{\beta}{2\left(p^{*}\right)} \psi(u) \int_{\mathbb{R}^{N}}|f(v)|^{2\left(p^{*}\right)} d x,
$$

where $\psi(u)=\tau(\|u\|)$ and $\tau: \mathbb{R}^{+} \rightarrow[0,1]$ is a nonincreasing $C^{\infty}$ function such that $\tau(t)=1$ if $t \leq T_{0}$ and $\tau(t)=0$ if $t \geq T_{1}$. Thus,

$$
\tilde{J}(v) \geq \tilde{Q}(\|v\|)
$$

where

$$
\tilde{Q}(t):=\frac{1}{p} t^{p}-\alpha c_{1} t^{q / 2}-\beta c_{2} t^{p^{*}} \psi(t) .
$$

It is clear that $\tilde{J}(v) \in C^{1}$ and is bounded from below. Moreover, we can follow from Proposition 3.3 and (3.7) that:

Proposition 3.5. (i) If $\tilde{J}(v)<0$, then $\|v\| \leq T_{0}$ and $\tilde{J}(v)=J(v)$;

(ii) for any $\beta>0$, there exists $\alpha_{3}>0$ such that if $0<\alpha<\alpha_{3}$ and $c<0$, then $\tilde{J}$ satisfies $(\mathrm{PS})_{c}$.

(iii) for any $\alpha>0$, there exists $\beta_{3}>0$ such that if $0<\beta<\beta_{3}$ and $c<0$, then $\tilde{J}$ satisfies $(\mathrm{PS})_{c}$.

Lemma 3.3. Denote $K_{c}:=\left\{v \in D^{1, p}\left(\mathbb{R}^{N}\right): \tilde{J}^{\prime}(v)=0, \tilde{J}(v)=c\right\}$. Then for any $m \in \mathbb{N}$, there is $\varepsilon_{m}<0$ such that $\gamma\left(\tilde{J}^{\varepsilon_{m}}\right) \geq m$.

Proof. Denote by $D_{0}^{1, p}(\Omega)$ the closure of $C_{0}^{\infty}(\Omega)$ with respect to the norm $\|u\|=\left(\int_{\Omega}|\nabla u|^{p} d x\right)^{1 / p}$. Extending functions in $D_{0}^{1, p}(\Omega)$ by 0 outside $\Omega$ we can assume that $D_{0}^{1, p}(\Omega) \subset D^{1, p}\left(\mathbb{R}^{N}\right)$. Let $X_{m}$ be a $m$-dimensional subspace of $D_{0}^{1, p}(\Omega)$. For any $v \in X_{m}, v \neq 0$, we write $v=r_{m} \omega$ with $\omega \in X_{m}$ and $\|\omega\|=1$. From the assumptions of $k(x)$, it is easy to see for every $\omega \in X_{m}$ with $\|\omega\|=1$ that there exist $d_{m}>0$ such that

$$
\int_{\Omega} k(x)|\omega|^{\frac{q}{2}} d x \geq d_{m}
$$

Thus for $0<r_{m}<T_{0}$, since all the norms are equivalent and $0<|\Omega|<+\infty$, by $\left(f_{4}\right)$ we have

$$
\begin{aligned}
& \tilde{J}(v)=J(v) \\
= & \frac{1}{p} \int_{\Omega}|\nabla v|^{p} d x-\frac{\alpha}{q} \int_{\Omega} k(x)|f(v)|^{q} d x-\frac{\beta}{2\left(p^{*}\right)} \int_{\Omega}|f(v)|^{2 \cdot p^{*}} d x \\
\leq & \frac{1}{p} \int_{\Omega}|\nabla v|^{p} d x-\frac{\alpha}{q} \int_{\Omega} k(x)\left(c|v|^{q / 2}-\varepsilon_{1}\right) d x-\frac{\beta}{2\left(p^{*}\right)} \int_{\Omega}\left(c|v|^{p *}-\varepsilon_{2}\right) d x
\end{aligned}
$$




$$
\begin{aligned}
& \leq \frac{1}{p} \int_{\Omega}|\nabla v|^{p} d x-\frac{\alpha c}{q} \int_{\Omega} k(x)|v|^{\frac{q}{2}} d x-\frac{\beta c}{2\left(p^{*}\right)} \int_{\Omega}|v|^{p^{*}} d x+\varepsilon \\
& \leq \frac{1}{p} r_{m}^{p}-\alpha c d_{m} r_{m}^{q / 2}-\beta c r_{m}^{p^{*}}+\varepsilon:=\varepsilon_{m}
\end{aligned}
$$

where $\varepsilon_{1}, \varepsilon_{2}$, and $\varepsilon>0$ are sufficiently small. Therefore, we can choose $r_{m} \in$ $\left(0, T_{0}\right)$ so small that $\tilde{J}(v) \leq \varepsilon_{m}<0$. Let $S_{r_{m}}=\left\{u \in D^{1, p}\left(\mathbb{R}^{N}\right):\|v\|=r_{m}\right\}$. Then $S_{r_{m}} \cap X_{m} \subset \tilde{J}^{\varepsilon_{m}}$. Hence, by Proposition 3.4(2), $\gamma\left(\tilde{J}^{\varepsilon_{m}}\right) \geq \gamma\left(S_{r_{m}} \cap X_{m}\right) \geq$ $m$.

Therefore, if we denote $\Gamma_{m}=\{A \in \Sigma: \gamma(A) \geq m\}$ and let

$$
c_{m}:=\inf _{A \in \Gamma_{m}} \sup _{v \in A} \tilde{J}(v)
$$

then

$$
-\infty<c_{m} \leq \varepsilon_{m}<0, \quad m \in \mathbb{N},
$$

because $\tilde{J}^{\varepsilon_{m}} \in \Gamma_{m}$ and $\tilde{J}$ is bounded from below.

Proposition 3.6. Let $\alpha, \beta$ be as in Proposition 3.5(ii) or (iii). Then all $c_{m}$ given by (3.8) are critical values of $\tilde{J}$ and $c_{m} \rightarrow 0$ as $m \rightarrow \infty$.

Proof. It is clear that $c_{m} \leq c_{m+1}$. By (3.9), $c_{m}<0$. Hence $c_{m} \rightarrow \bar{c} \leq 0$. Moreover, since $(\mathrm{PS})_{c}$ is satisfied, it follows from a standard argument (e.g. [17]) that all $c_{m}$ are critical values of $\tilde{J}$. Now, we claim that $\bar{c}=0$. If $\bar{c}<0$, because $K_{\bar{c}}$ is compact and $K_{\bar{c}} \in \Sigma$, it follows from that $\gamma\left(K_{\bar{c}}\right)=m_{0}<+\infty$ and there exists $\delta>0$ such that $\gamma\left(K_{\bar{c}}\right)=\gamma\left(N_{\delta}\left(K_{\bar{c}}\right)\right)=m_{0}$. By the deformation lemma, there exist $\epsilon>0(\bar{c}+\epsilon<0)$ and an odd homeomorphism $\zeta$ such that

$$
\zeta\left(\tilde{J}^{\bar{c}+\epsilon} \backslash N_{\delta}\left(K_{\bar{c}}\right)\right) \subset \tilde{J}^{\bar{c}-\epsilon} .
$$

Since $c_{m}$ is increasing and converges to $\bar{c}$, there exists $m \in \mathbb{N}$ such that $c_{m}>\bar{c}-\epsilon$ and $c_{m+m_{0}} \leq \bar{c}$. There exists $A \in \Gamma_{m+m_{0}}$ such that $\sup _{u \in A} \widetilde{G}(u)<\bar{c}+\epsilon$. By Proposition 3.4, we have

$$
\gamma\left(\overline{A \backslash N_{\delta}\left(K_{\bar{c}}\right)}\right) \geq \gamma(A)-\gamma\left(N_{\delta}\left(K_{\bar{c}}\right)\right) \geq n, \quad \gamma\left(\zeta\left(\overline{A \backslash N_{\delta}\left(K_{\bar{c}}\right)}\right)\right) \geq n .
$$

Therefore,

Consequently

$$
\zeta\left(\overline{A \backslash N_{\delta}\left(K_{\bar{c}}\right)}\right) \in \Gamma_{n}
$$

$$
\sup _{u \in \zeta\left(\overline{A \backslash N_{\delta}\left(K_{\bar{c}}\right)}\right)} \widetilde{G}(u) \geq c_{n}>\bar{c}-\epsilon .
$$

On the other hand, by (3.10) and (3.11),

$$
\zeta\left(A \backslash N_{\delta}\left(K_{\bar{c}}\right)\right) \subset \zeta\left(\tilde{J}^{\bar{c}+\varepsilon} \backslash N_{\delta}\left(K_{\bar{c}}\right)\right) \subset \tilde{J}^{\bar{c}},
$$

which contradicts (3.12). Hence $c_{n} \rightarrow 0$.

Proof of Theorem 2.3. By Proposition 3.5(i), $\tilde{J}(v)=J(v)$ if $\tilde{J}<0$. This and Proposition 3.6 give the result. 
Proof of Theorem 1.1. The result follows from Theorem 2.1 since $u_{m}=f\left(v_{m}\right)$ $\neq u_{n}=f\left(v_{n}\right)$ if $v_{m} \neq v_{n}$.

\section{References}

[1] M. J. Alves, P. C. Carrião, and O. H. Miyagaki, Non-autonomous perturbations for a class of quasilinear elliptic equations on R, J. Math. Anal. Appl. 344 (2008), no. 1, 186-203.

[2] J. G. Azorero and I. P. Alonso, Multiplicity of solutions for elliptic problems with critical exponent or with a nonsymmetric term, Trans. Amer. Math. Soc. 323 (1991), no. 2, 877-895.

[3] L. Bocardo and F. Murat, Almost everywhere convergence of the gradients of solutions to elliptic and parabolic equations, Nonlinear Anal. 19 (1992), no. 6, 581-597.

[4] J. Chabrowski, Concentration-compactness principle at infinity and semilinear elliptic equations involving critical and subcritical Sobolev exponents, Calc. Var. Partial Differential Equations 3 (1995), no. 4, 493-512.

[5] M. Colin and L. Jeanjean, Solutions for a quasilinear Schrödinger equations: a dual approach, Nonlinear Anal. 56 (2004), no. 2, 213-226.

[6] M. A. Krasnoselskii, Topological Methods in the Theory of Nonlinear Integral Equations, Pergamon, Elmsford, NY, 1964.

[7] S. Kurihura, Large amplitude quasi-solitons in superfluid films, J. Phys. Soc. Japan 50 (1981), 3262-3267.

[8] P. L. Lions, The concentration-compactness principle in the calculus of variations. The locally compact case, part 1, 2, Ann. Inst. H. Poincaré Anal. Non Linéaire 1 (1984), no. 2, 109-145; Ann. Inst. H. Poincaré Anal. Non Linéaire 1 (1984), no. 4, 223-283.

[9] J. Q. Liu and Z. Q. Wang, Soliton solutions for quasilinear Schrödinger equations. I, Proc. Amer. Math. Soc. 131 (2003), no. 2, 441-448.

[10] J. Q. Liu, Y. Q. Wang, and Z. Q. Wang, Soliton solutions to quasilinear Schrödinger equations. II, J. Differential Equations 187 (2003), no. 2, 473-493.

[11] Solutions for quasilinear Schrödinge equations via the Nehari method, Comm. Partial Differential Equations 29 (2004), no. 5-6, 879-901.

[12] A. Moameni, Existence of soliton solutions for a quasilinear Schrödinger quation involving critical exponent in $R^{N}$, J. Differential Equations 229 (2006), no. 2, 570-587.

[13] A. Nakamura, Damping and modification of exciton solitary waves, J. Phys. Soc. Japan 42 (1977), 1824-1835.

[14] J. M. do Ó, O. Miyagaki, and H. Olimpio, Soliton solutions for quasilinear Schrodinger equations with critical growth, J. Differential Equations 248 (2010), no. 4, 722-744.

[15] I. Peral, Multiplicity of solutions for the p-Laplacian, in: A. Ambrosetti, K. C. Chang, I. Eklend (Eds.), Lecture Notes at the Second School on Nonlinear Functional Analysis and Applications to Differential Equations at ICTP, Trieste, (1997), 153-202.

[16] M. Poppenberg, K. Schmitt, and Z. Q. Wang, On the existence of soliton solutions to quasilinear Schrödinger equations, Calc. Var. Partial Differential Equations 14 (2002), no. 3, 329-344.

[17] P. H. Rabinowitz, Minimax Methods in Critical Point Theory with Applications to Differential Equations, CBMS Regional Conf. Ser. in Math. No. 65, Amer. Math. Soc., Providence, RI, 1986.

[18] U. Severo, Existence of weak solutions for quasilinear elliptic equations involving the p-Laplacian, Electron. J. Differential Equations 2008 (2008), no. 56, 1-16.

[19] Elves A. B. Silva and Gilberto F. Vieira, Quasilinear asymptotically periodic Schrodinger equations with critical growth, Calc. Var. Partial Differential Equations 39 (2010), no. $1-2,1-33$. 
[20] Y. J. Wang, J. Yang, and Y. M. Zhang, Quasilinear elliptic equations involving the $N$-Laplacian with critical exponential growth in $\mathbb{R}^{N}$, Nonlinear Anal. 71 (2009), no. 12, 6157-6169.

[21] Y. J. Wang, Y. M. Zhang, and Y. T. Shen, Multiple solutions for quasilinear Schrodinger equations involving critical exponent, Appl. Math. Comp. 216 (2010), no. 3, 849-856.

[22] M. Willem, Minimax Theorems, Birkhäuser, Boston, 1996.

Department of Mathematical Sciences

TSinghua University

BeiJing 100084, P. R. China

E-mail address: yjwang@math.tsinghua.edu.cn 